\title{
DETERMINATION OF THE FINITE QUATERNARY LINEAR
}

\author{
GROUPS* \\ BY \\ HOWARD H. MITCHELL
}

\section{§1. Introduction.}

A number of writers have concerned themselves with the finite collineation groups in ordinary three-dimensional space, among them JoRDAN, KLEIN, MASChKE, and WitTing. $\dagger$ A complete enumeration of such groups however was first given by BLICHFELDT. $\ddagger$ His determination of the groups was accomplished largely by means of theorems obtained by him in two previous papers. $\S$ His methods were mainly algebraic, the irreducibility of certain polynomials in given domains playing an important rôle.

About the same time a determination of those groups which contain homologies was given by BAGNERA.\| The methods employed by him were of geometrical nature, the possible configurations formed by the centers and axial planes of the homologies being considered in detail.

It is the purpose of the present writer to make the determination of all the groups, which do not contain homologies, by geomethical methods, making use of well-known properties of the linear complex, the quadric, etc. The determination applies equally well to the quaternary groups in which the transformations are those of a general modular space, $\{$ provided the orders of none of the transformations be divisible by the modulus. Bagnera's methods apply also to such groups which contain homologies. A large class of quaternary groups which are determined by the $G F(p)$ and which do contain transformations of period divisible by the modulus have been com-

* Presented to the Society October 28, 1911.

$\dagger$ For a résumé of the work done in this field consult Wiman, Endliche Gruppen linearer Substitutionen, Encyklopadie der Mathematischen Wissenschaften, vol. I, pp. 522-554.

† $\mathrm{M}$ a the matis che Annalen, vol. 60 (1905), pp. 204-231.

8 These Tra n s a c tio n s, vol. 4 (1903), pp. 387-397, vol. 5 (1904), pp. 310-325.

\|Rendicontidel Circolo Matematicodi Palermo, vol. 19 (1905), pp. 1-56.

T C. Vhblen and Bussey, Finite Projective Geometries, these Tra n a a t i o n s vol. 7 (1906), pp. 241-259. 
pletely enumerated by Dickson.* These papers therefore supplement each other. In the present paper some special groups are noticed incidentally whose orders are divisible by the modulus.

Following BLICHFELDT, we shall apply the term "primitive" to those groups which do not leave invariant a point, a plane, two skew lines, or a tetrahedron. One class of such groups has an invariant quadric surface, $\dagger$ and another class an invariant linear complex. The others are certain groups $f$ having an invariant $G_{16}$, and three simple groups of order 25920, 2520, and 168. The first of these three was found by WrTring. $\S$ It contains homologies of period 3 and hence is in Bagnera's list. The groups which are holoedrically isomorphic with either a symmetric or an alternating group are of orders $\frac{1}{2} 7 !, 6 !, \frac{1}{2} 6 !, 5 !$ (two types), $\frac{1}{2} 5 !$ (two types). Those of order $6 !$ and $\frac{1}{2} 6$ ! have an invariant linear complex, and those of order $5 !$ and $\frac{1}{2} 5 !$ either an invariant complex or an invariant quadric. The one of order 5! which has an invariant quadric contains homologies of period 2.

\section{\$2. Groups which contain homologies.}

Bagnera's results in the case of the groups containing homologies may be summarized in the following theorems. For the proofs and for a detailed discussion of the allied geometrical configurations the reader is referred to his paper.

Theorem 1. No primitive group contains homologies of period greater than 3 .

Theorem 2. The only primitive group which contains homologies of period 3 is of order 25920 .

Theorem 3. No primitive group contains two homologies of period 2, whose product is of period greater than 5 .

Theorem 4. Groups of order 120 and 288 are the only primitive groups which contain two homologies of period 2 , whose product is of period 3 , but none whose product is of higher period.

Theorem 5. Groups of order 576, 1152, 1920, and 11520 are the only primitive groups which contain two homologies of period 2 , whose product is of period 4, but none whose product is of higher period.

Theorem 6. A group of order 7200 is the only primitive group which contains two homologies of period 2, whose product is of period 5.

* American Journal of Mathematics, vol. 28 (1906), pp. 1-16.

† These groups were first determined by Goursat, Annales s cientifiques de

l'E cole Norma le Su périeure, Ser. 3, vol. 6 (1889), pp. 9-102.

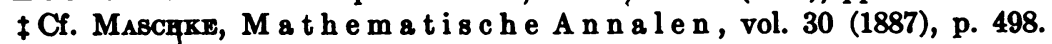

Insugural-Dissertation, Göttingen (1887), p. 27. Cf. also MAscrexe, M a the matische Annalen, vol. 33 (1889), p. 320 . 
§3. General properties of groups containing skew perspectivities.

In the discussion of groups containing skew perspectivities the following facts will be found of use:

Two skew perspectivities must leave invariant in common at least two skew lines, since their product can leave invariant no point unless on a line left invariant by both.

Two skew perspectivities cannot have one axis in common.

If an axis of one skew perspectivity intersects an axis of a second, the other axis of the first must intersect the other axis of the second.

If an axis of one skew perspectivity meets both axes of a second, the other axis of the first must meet both axes of the second.

A linear complex is left invariant by any skew perspectivity whose axes are in the complex.

If a skew perspectivity leaves a linear complex invariant, either its axes are lines of the complex or else it is of period 2 and its axes are axes of a congruence contained by the complex.

Two skew reflections whose axes are axes of congruences in the same linear complex must leave invariant in common all the lines of a regulus. (If the two congruences contained a flat pencil in common the product of the reflections would be an elation in that plane.)

Theorem 7. In a primitive group a subgroup generated by skew perspectivities of higher period than 2 whose axes lie in a congruence will be contained by a group leaving a single linear complex invariant.

In a primitive group there will be skew perspectivities conjugate with those whose axes are in the congruence which do not leave the congruence invariant. Such a skew perspectivity must generate with any skew perspectivity whose axes lie in the congruence and whose axes its axes do not intersect either a group leaving invariant all the lines of a regulus or a group leaving a congruence invariant. In the latter case however each skew perspectivity will be contained by more than one group which does leave invariant all the lines of a regulus, and, since the new congruence cannot have more than one regulus in common with the original congruence, there will also be in this case skew perspectivities which do not leave the congruence invariant and which generate with skew perspectivities whose axes do lie in the congruence a group leaving invariant all the lines of a regulus. The axes of such a skew perspectivity would determine a complex together with the congruence.

The truth of these statements follows from the consideration of the various cases which can arise. Two skew perspectivities of period greater than 5, whose axes do not intersect, can generate no group. If they are of period 5, they must generate a group of order 60 , which leaves invariant all the lines 
of a regulus, or else a group of order $2 \cdot 60 \cdot 60$ in which every skew $C_{5}$ lies in five such $G_{60}$. If they are of period 4 , they must generate a group of order 24 leaving invariant all the lines of a regulus. If they are of period 3 , they must generate a group of order $12,60,2 \cdot 12 \cdot 12,2 \cdot 12 \cdot 60$. The group of order 12 leaves invariant all the lines of a regulus; that of order 60 may do so, but if not each $C_{3}$ lies in two $G_{12}$ which do; in the groups of order $2 \cdot 12 \cdot 12$ and $2 \cdot 12 \cdot 60$ each $C_{3}$ lies in several such $G_{12} .^{*}$

Theorem 8. A group leaving invariant a linear complex but not a congruence cannot contain a transformation of even period greater than 4 having a single line of fixed points.

A power of such a transformation must be a reflection whose axes are axes of a congruence contained by the complex. Any reflection conjugate with this one must leave invariant all the lines of a regulus in common with it and such a regulus will contain two lines left invariant by the transformation having a line of fixed points. Hence, since that transformation is of period not less than 6 , any reflection conjugate with it must interchange its axes. But there will be conjugate reflections interchanging those axes which are not themselves commutative.

\section{§4. Groups containing skew perspectivities of period greater than 3 .}

Theorem 9. There is no primitive group which contains skew perspectivities of period greater than 5 .

Two such skew perspectivities which are not commutative can generate no group.

Theorem 10. No primitive group which does not leave a quadric invariant can contain skew perspectivities of period 5 .

Two skew perspectivities of period 5 which are not commutative must generate a group of order 60 if they leave invariant all the lines of a regulus, a group of order $2 \cdot 5 \cdot 60$ if their axes intersect along one line, or a group of order $2 \cdot 60 \cdot 60$. Each of the last two groups contains transformations of period 10 which leave fixed all the points on one axis of the congruence and are of period 5 on the other. Hence no primitive group can contain them (Theorems 7, 8).

Hence any two skew perspectivities of period 5 which are not commutative must leave invariant all the lines of a regulus. But if a quadric does not remain invariant, not all the skew perspectivities of period 5 which do not lie in a particular $G_{60}$ will be commutative with the $G_{60}$. Two $G_{60}$ having a skew $C_{5}$ in common will leave invariant a congruence and hence must generate a group of order $2 \cdot 60 \cdot 60$.

\footnotetext{
* For a more detailed discussion of the imprimitive groups the reader may consult BucHFELDT, these Tran s a c t i n s, vol. 6 (1905), pp. 230-236.
} 
Theorem 11. A $G_{16.120}$ containing an invariant $G_{16}$ is the only primitive group containing skew perspectivities of period 4, but no homologies, which does not leave a quadric invariant.

Two skew perspectivities of period 4 which are not commutative must generate either a group of order $2 \cdot 4 \cdot 12$ (if their axes intersect along one line) or a group of order 24 leaving invariant all the lines of a regulus. A group of the former type contains $C_{6}$ which leave fixed all the points on one axis of the congruence and are of period 3 on the opposite axis. Hence it cannot be contained by a primitive group (Theorems 7,8 ).

Hence any two skew perspectivities of period 4 which are not commutative must generate a group of order 24 leaving invariant all the lines of a regulus. A $G_{24}$ is generated by

$$
\begin{aligned}
{\left[x_{1}, i x_{2}, i x_{3}, x_{4}\right] } & \\
x_{1}^{\prime}-x_{2}^{\prime}=x_{1}-x_{2}, \quad x_{3}^{\prime}-x_{4}^{\prime} & =x_{3}-x_{4}, \\
x_{1}^{\prime}+x_{2}^{\prime}=i\left(x_{1}+x_{2}\right), & x_{3}^{\prime}+x_{4}^{\prime}=i\left(x_{3}+x_{4}\right) .
\end{aligned}
$$

If no quadric remains invariant, not all the other skew perspectivities of period 4 will be commutative with all of those in this $G_{24}$. Consider one which is not. Since it must leave invariant all the lines of a regulus in common with a skew $C_{4}$ with which it is not commutative, it will leave invariant a congruence in common with the above $G_{24}$. Since we cannot have two skew $C_{4}$ whose axes intersect along a single line, this $C_{4}$ must be commutative with a $C_{4}$ in the original $G_{24}$. Hence every $C_{4}$ must be commutative with one or all three of the $C_{4}$ in that $G_{24}$. There cannot be three mutually commutative $C_{4}$ without involving homologies of period 2 . Hence each of the $C_{4}$ in the original $G_{24}$ can be commutative with but three $C_{4}$ and one of these will be commutative with the $G_{24}$. There can be but $3+3 \cdot 2+1=10 C_{4}$ altogether. Two of the others we take to be

$$
\begin{gathered}
{\left[x_{1}, i x_{2}, x_{3}, i x_{4}\right] ;} \\
x_{1}^{\prime}-x_{4}^{\prime}=x_{1}-x_{4}, \quad x_{1}^{\prime}+x_{4}^{\prime}=i\left(x_{1}+x_{4}\right), \\
x_{2}^{\prime}-x_{3}^{\prime}=x_{2}-x_{3}, \quad x_{2}^{\prime}+x_{3}^{\prime}=i\left(x_{2}+x_{3}\right) .
\end{gathered}
$$

A group is generated of order $16 \cdot 120$, leaving invariant the $G_{16}$ which contains the squares of all the ten $C_{4}$. The axes of these ten reflections are lines of the invariant complex. The axes of the other five reflections in the $G_{16}$ are axes of congruences which belong to that complex.

\section{§5. Groups containing sleew perspectivities of period 3 .}

Theorem 12. The only primitive groups which contain skew perspectivities of period 3 whose axes intersect along a single line, but which contain no skew 
perspectivities of higher period and no homologies, and which do not leave a quadric invariant, are of order $16 \cdot 60$ and $16 \cdot 360$, each having an invariant $G_{16}$.

Two skew perspectivities of period 3 which are not commutative must generate a group of one of the following orders: $12,60,2 \cdot 3 \cdot 4,2 \cdot 3 \cdot 12$, $2 \cdot 3 \cdot 60,2 \cdot 12 \cdot 12,2 \cdot 12 \cdot 60$. We may suppose that the $G_{12}$ is the only group which leaves invariant all the lines of a regulus, since a $G_{60}$ having that property would contain skew perspectivities of period 5 . All the above groups except those of order $12,60,2 \cdot 3 \cdot 4$ contain $C_{6}$ which leave invariant all the points on one axis of the congruence and are of period three on the other axis. Such groups can be contained by no primitive group (Theorems $7,8)$.

Consider a group of order $2 \cdot 3 \cdot 4$ generated by two $C_{3}$ whose axes intersect along a single line. It contains $C_{4}$ which leave fixed all the points on one axis of the congruence and are of period 2 on the other. This group must be contained by a group leaving a linear complex invariant (Theorem 7). In such a group every reflection conjugate with the invariant reflection of the above group must leave invariant all the lines of a regulus in common with it. If the product of two such reflections were a skew $C_{3}$, there would be on two of the lines of the invariant regulus a $C_{4}$ and a $C_{3}$ such that the square of the $C_{4}$ would transform the $\mathrm{C}_{3}$ into its inverse. But there is no such group on a line. Hence any two such reflections must interchange each other's axes. The product of two such reflections moreover must be a reflection whose axes are lines of the complex.

We find readily that there must be five reflections of this sort, which we may take to be $\left[x_{1}, x_{2},-x_{3},-x_{4}\right],\left[x_{4}, x_{3},-x_{2},-x_{1}\right],\left[x_{4},-x_{3},-x_{2}, x_{1}\right]$, $\left[x_{3}, x_{4}, x_{1}, x_{2}\right],\left[-x_{3}, x_{4}, x_{1},-x_{2}\right]$. The fifth lies in the $G_{16}$ generated by the first four. The whole group leaving invariant the $G_{16}$ and the complex is of order $16 \cdot 120$ and it contains skew perspectivities of period 4 . The group in this case must be of order $16 \cdot 60$.

We inquire what groups can contain the $G_{960}$. The skew perspectivitles whose axes lie in one of the five congruences generate a $G_{96}$. We may show that such a $G_{96}$ must be contained by at least one other $G_{960}$. For consider a skew $C_{3}$ not in the $G_{960}$. It must generate with a $C_{3}$ in the $G_{96}$ whose axes its axes do not intersect either a $G_{12}$ or a $G_{60}$. In the latter case the $C_{3}$ in the $G_{96}$ will be contained by two $G_{12}$ and since the $G_{60}$ can be generated by these two $G_{12}$ not more than one of them can lie in the $G_{960}$. A $G_{12}$ not in the $G_{900}$ which contains a skew $C_{3}$ in the $G_{96}$ will leave a linear complex invariant in common with the $G_{96}$ and hence must generate with the latter group another $G_{960}$.

We will show that another $G_{960}$ containing the $G_{96}$ must leave invariant the same $G_{16}$. Hence it will follow that every skew $C_{3}$ which generates a $G_{12}$ 
with a $C_{3}$ in the $G_{96}$ will leave invariant that $G_{10}$. Since a $G_{80}$ may be generated by the two $G_{12}$ containing a $C_{3}$, it must also follow that every $C_{3}$ which generates a $G_{80}$ with a $C_{3}$ in the $G_{80}$ must leave invariant the same $G_{16}$. Hence that $G_{10}$ must remain invariant under any larger group.

We proceed to show that any $G_{860}$ containing the $G_{90}$ must leave invariant the same $G_{16}$. Consider the $G_{96}$ with fixed lines $x_{1}=0, x_{2}=0$ and $x_{3}=0$, $x_{4}=0$. It leaves invariant three $G_{8}$ which contain no $C_{4}$ according as the three pairs of points $(1000)(0100),(1100)(1-100),(1 i 00)(1-i 00)$ are joined by the axes of the reflections to $(0001)(0010),(0011)(00-11)$, (00i1) $(.00-i 1)$ respectively, or to those three pairs permuted in a cyclic order. If more than one of these $G_{8}$ is contained by a $G_{16}$ we will have as the product of reflections which interchange $x_{1}=0, x_{2}=0$ and $x_{3}=0, x_{4}=0$ and which lie in different $G_{16}$ a transformation which is of period 3 on each of those lines and on the three $G_{8}$. This transformation may be of period 6 , but its square must be of period 3 . This square must be commutative with one of the $C_{3}$ in the $G_{98}$. In the $G_{9}$ generated there must be either an homology of period 3 or a skew perspectivity of period 3 whose axes are in the congruence and which is not in the $G_{96}$. Either case is impossible. Hence the $G_{980}$ which contain the $G_{96}$ must all leave invariant the same $G_{16}$. The group generated must be of order $16 \cdot 360$.

Theorem 13. The only primitive groups which contain skew perspectivities of period 3 , but none whose axes intersect by pairs, no skew perspectivities of higher period, and no homologies, and which do not leave a quadric invariant are holoedrically isomorphic respectively with the $G_{360}^{6}$, the $G_{720}^{6}$, and the $G_{2580}^{7}$.

Any two $G_{12}$ having a $C_{3}$ in common will leave invariant a congruence and hence must generate a $G_{60}$. There must be at least three $G_{12}$ containing a $C_{3}$ provided a congruence does not remain invariant under the group. Hence a $G_{12}$ must lie in at least two $G_{80}$. A particular reflection $R$ in the $G_{12}$ will interchange the axes of two $C_{3}$ in each $G_{80}$. Let the two $C_{3}$ of this sort in one $G_{60}$ be denoted by $A$ and $A^{\prime}$, and the two in the other by $B$ and $B^{\prime}$

We will show that $A$ and $B$ cannot generate a $G_{60}$. For suppose this is the case. $R$ would then leave invariant each of the fixed lines of the $G_{80}$, for the axes of $R, A$, and $B$ would lie in a congruence determined by the two axes of $R$, one axis of $A$, and one axis of $B$. Hence either $R$ would lie in the $G_{60}$ or else its product by the $C_{2}$ in the $G_{80}$ which interchanges the same two pairs of axes would be a $C_{2}$ commutative with the $G_{00}$.

Consider the first case. $R$ can lie in but one $G_{12}$ leaving invariant all the lines of a regulus. For two such $G_{12}$ would leave invariant a congruence and hence would have to generate a $G_{60}$. But a $C_{2}$ in a $G_{60}$ lies in but one $G_{12}$. Hence the $G_{60}$ generated by $A$ and $B$ would have to contain the $G_{12}$ which contains $R$. But the $G_{12}$ and $A$ generate a $G_{\infty}$ not containing $B$.

Trans. Am. Math. 800. 9 
Consider the second case. Since there can be but one $G_{12}$ containing $R$, the reflection commutative with $R, A$, and $B$ must leave the $G_{12}$ invariant. If it is commutative with the $G_{12}$ it cannot be commutative with both $A$ and $B$. It must then be the product of $R$ and a reflection commutative with the $G_{12}$. It must then be left invariant by the $G_{4}$ of the $G_{12}$. It must then be left invariant by the $G_{60}$ generated by $A$ and this $G_{4}$. Consequently it must be left invariant by the $G_{12}$. But this is impossible unless it is commutative with the $G_{12}$.

Hence $A$ and $B$ cannot generate a $G_{60}$. Similarly $A$ and $B^{\prime}$ cannot generate a $G_{80}$. It may be shown that $A$ cannot generate a $G_{12}$ with $B$ and another $G_{12}$ with $B^{\prime}$. For in this case $A, B$, and $B^{\prime}$ would have to generate a $G_{80}$. But this is impossible since $B$ and $B^{\prime}$ generate a $G_{60}$ not containing $A$. Hence $A$ must be commutative with either $B$ or $B^{\prime}$, say with $B^{\prime}$. Since $A$ cannot be commutative with both $B$ and $B^{\prime}$, it must generate a $G_{12}$ with $B$.

We take these three $C_{3}$ as follows:

$$
\begin{array}{rlrl}
A:\left[\omega x_{1}, \omega x_{2},\right. & \left.\omega^{2} x_{3}, \omega^{2} x_{4}\right] & \left(\omega^{2}+\omega+1=0\right) ; \\
B: x_{1}^{\prime}-x_{4}^{\prime} & =\omega\left(x_{1}-x_{4}\right), & x_{2}^{\prime}-x_{3}^{\prime} & =\omega\left(x_{2}-x_{3}\right), \\
x_{1}^{\prime}+2 x_{4}^{\prime} & =\omega^{2}\left(x_{1}+2 x_{4}\right), & x_{2}^{\prime}+2 x_{3}^{\prime} & =\omega^{2}\left(x_{2}+2 x_{3}\right) ; \\
B_{1}^{\prime}-x_{2}^{\prime} & =\omega\left(x_{1}-x_{2}\right), & x_{3}^{\prime} & =\omega^{2} x_{3}, \\
B^{\prime}: & x_{4}^{\prime} & =\omega x_{4} .
\end{array}
$$

In order that $B$ and $B^{\prime}$ shall generate a $G_{60}$ we must have $\left(B^{\prime} B\right)^{5}=I$. The condition for this is $a=-1$. $^{*}$ The reflection interchanging all three pairs of axes is given by

$$
R: \begin{array}{ll}
x_{1}^{\prime}=x_{3}+x_{4}, & x_{3}^{\prime}=-\frac{1}{2} x_{1}+\frac{1}{2} x_{2}, \\
x_{2}^{\prime}=-x_{3}+x_{4}, & x_{4}^{\prime}=-\frac{1}{2} x_{1}-\frac{1}{2} x_{2} .
\end{array}
$$

We then take $E_{1}=A, E_{2}=A^{2} B A^{2}, E_{3}=R B^{\prime}, \dagger E_{4}=R$. It may be shown that $I=E_{1}^{3}=E_{2}^{2}=E_{3}^{2}=E_{4}^{2}=\left(E_{1} E_{2}\right)^{3}=\left(E_{1} E_{3}\right)^{2}=\left(E_{1} E_{4}\right)^{2}=\left(E_{2} E_{3}\right)^{2}$ $=\left(E_{2} E_{4}\right)^{2}=\left(E_{3} E_{4}\right)^{3}$. These operators therefore generate a group simply isomorphic with the $G_{360}^{6} . \neq$

Under the $G_{360}$ the $G_{12}$ generated by $E_{1}$ and $E_{2}$ is left invariant by a $G_{24}$. Hence if the $G_{800}$ is self-conjugate under a larger group, there must be skew perspectivities commutative with that $G_{12}$. The only such skew perspectivity which interchanges the axes of $B^{\prime}$ is the reflection $\left[-x_{2}, x_{1}, x_{4},-x_{2}\right]$.

* This may be obtained from the fact that the coefficient of $\rho^{2}$ in the characteristic equation of $B^{\prime} B$ must be unity. The coefficient of $\rho^{2}$ is -1 for any value of $a$.

$\dagger$ In a product the order of operating is taken from right to left.

†. H. Moore, Proceeding o f the Lond on Mathematical Society, vol. 28 (1897), pp. 357-366. Also Dicksos, Linear Groups, p. 289. 
Hence the $G_{300}$ is self-conjugate under a group of order 720 , which must be simply isomorphic with the $G_{720}^{6}$.

The $G_{380}$ and the $G_{720}$ leave invariant a linear complex, i. e., that determined by the axes of $A$ and $B^{\prime}$ and one axis of $B$.

In a group containing the $G_{360}$ not self-conjugately there must be more $C_{3}$. Hence a $C_{3}$ must lie in at ieast four $G_{12}$ and $4 \cdot 3 / 1 \cdot 2 G_{60}$. Hence $R$ must interchange the axes of two other reflections, $C$ and $C^{\prime}$, which together generate a $G_{80}$. One of them, say $C^{\prime}$, must be commutative with $A$. Similarly $C^{\prime}$ must be commutative with either $B$ or $B^{\prime}$. If it is commutative with $B^{\prime}$ homologies of period 3 will be present. Hence it must be commutative with $B$. We then have

$$
\begin{aligned}
x_{1}^{\prime}-b x_{2}^{\prime} & =\omega\left[x_{1}-b x_{2}\right], \\
C^{\prime}: \quad(b-1) x_{1}^{\prime}-(b+1) x_{2}^{\prime} & =\omega^{2}\left[(b-1) x_{1}-(b+1) x_{2}\right], \\
x_{4}^{\prime}-b x_{3}^{\prime} & =\omega\left[x_{4}-b x_{3}\right], \\
(b-1) x_{4}^{\prime}-(b+1) x_{3}^{\prime} & =\omega^{2}\left[(b-1) x_{4}-(b+1) x_{3}\right] .
\end{aligned}
$$

In order that $B^{\prime}$ and $C^{\prime}$ shall generate a $G_{12}$ we may take $\left(B^{r^{-1}} C^{\prime}\right)^{2}=I$. The condition for this is $b^{2}+b+2=0$. We put $E_{5}=E_{4} C^{\prime}$ and find $I=E_{5}^{2}=\left(E_{1} E_{5}\right)^{2}=\left(E_{2} E_{5}\right)^{2}=\left(E_{3} E_{5}\right)^{2}=\left(E_{4} E_{5}\right)^{3 *}$. Hence $E_{5}$ generates with the $G_{360}$ a group simply isomorphic with the $G_{2520}^{7} \cdot \dagger$

If the $G_{2520}$ is contained self-conjugately by a larger group, the $G_{360}$ must be contained self-conjugately by a larger group. The only such group is the $G_{720}$. Since $C^{\prime}$ is the only $C_{3}$ in the $G_{2520}$ which is commutative with the $G_{12}$ generated by $E_{1}$ and $E_{2}$, its axes must be interchanged by [ $-x_{2}, x_{1}, x_{4},-x_{3}$ ]. But this is not the case. $\ddagger$ Hence the $G_{2520}$ is self-conjugate under no larger group.

If the $G_{2520}$ is contained not self-conjugately by a larger group, a $C_{3}$ must be contained by at least five $G_{12}$ and $5 \cdot 4 / 1 \cdot 2 G_{60}$. There must then be two other $C_{3}, D$ and $D^{\prime}$, whose axes are interchanged by $R$, and one of which, say $D^{\prime}$, is commutative with $A$. Since $D^{\prime}$ cannot be commutative with $B^{\prime}$ without involving homologies of period 3 , it must be commutative with $B$. It must then generate a $G_{12}$ with $B^{\prime}$. The only such $C_{3}$ are $C^{\prime}$ and the similar one to $C^{\prime}$ obtained by choosing the other value of $b$. The latter must then be $D^{\prime}$. But it does not generate a $G_{12}$ with $C^{\prime}$. Hence no group can contain the $G_{2520}$.

* These relations follow readily from the relations of the $G_{360}$ together with:

$$
C^{\prime 3}=I, \quad E_{4} C^{\prime} E_{4}=C^{\prime-1}, \quad E_{1} C^{\prime}=C^{\prime} E_{1}, \quad E_{2} C^{\prime}=C^{\prime} E_{2}, \quad\left(B^{\prime-1} C^{\prime}\right)^{2}=I .
$$

† E. H. Moore, l. c.; Dickson, l. c.

$\mp$ The axes of $C^{\prime}$ are interchanged by this $C_{2}$ if the coefficients of the transformations are reduced modulo 7. In this case a group exists simply isomorphic with the $G_{5040}^{7}$. 


\section{§6. Groups containing skew reflections which interchange each other's axes.}

Theorem 14. The only primitive groups leaving invariant a linear complex, which do not contain skew perspectivities of higher period than 2 , are of order $16 \cdot 5,16 \cdot 10,16 \cdot 20,60,120$.

We consider first those groups which contain reflections whose axes are axes of a congruence belonging to the invariant complex. Any two of these reflections must leave invariant all the lines of a regulus in common and hence must be commutative, since we are assuming that no skew perspectivities of higher period than 2 are present. Moreover the product of two of these reflections must be a reflection whose axes are lines of the complex. We find that the only possible primitive groups are groups permuting five of these reflections which are all in a $G_{16}$ (see the similar discussion under Theorem 12). The groups of this sort which contain no skew perspectivities of higher period than 2 are of order $16 \cdot 5,16 \cdot 10,16 \cdot 20$.

We suppose now that the group does not contain reflections whose axes are axes of a congruence belonging to the invariant complex. Hence the group can contain no four-group in which each reflection leaves fixed the axes of the other two, for in such a four-group the axes of one of the three reflections would be axes of a congruence. Consequently no transformation can leave fixed the axes of more than one reflection unless the axes of those reflections meet along a single line and the transformation commutative with them leaves fixed all the points on the other fixed line of the dihedral group generated by the reflections. The group leaving fixed all four vertices of a tetrahedron must be cyclic, since four of the lines of the tetrahedron must be lines of the complex and a transformation leaving fixed all the points on one of these lines must leave fixed all the points on the opposite line also. The two axes of the invariant congruence of the above group cannot be interchanged, for in that case there would be commutative transformations of the same period having different lines of fixed points, and hence the group would not be cyclic.

It will be shown later (Theorem 19) that there is no primitive group containing no commutative reflections. A reflection in the above group can be commutative with but one cyclic group having a line of fixed points, for otherwise it would be commutative with reflections which left fixed its axes. Hence all reflections commutative with any reflection in this group must leave invariant the two axes of the congruence. We shall then have three sets of reflections, those in two of the sets leaving fixed one pair of points and interchanging the other, and those in the third set interchanging both pairs of points.

The first two sets of reflections cannot be conjugate, since they are commutative with transformations of different periods. Consider a particular 
reflection of the first set. The product of this reflection and any reflection conjugate with those of the second set must be of even period. Hence any reflection of the latter type must be commutative with a reflection which is commutative with this particular reflection. But no more reflections can be commutative with a reflection in either the first or second set. Also not all the reflections in the third set are commutative with the particular reflection we are considering and hence no more reflections can be commutative with them. Hence all the reflections which are conjugate with those in the second set must be in the group leaving the congruence invariant. No primitive group is therefore possible.

If no transformation except the identity leaves fixed the axes of more than one reflection, and if the order of the group leaving both axes fixed be denoted by $d$, then that group will be self-conjugate under a group of order $2 d$ interchanging the axes. Hence, if $\Omega$ denotes the order of the whole group, there will be $\Omega /(2 d)$ conjugate reflections and $(d-1) \Omega /(2 d)$ transformations each leaving fixed one pair of axes.

A cyclic group of odd order, $d$, will be self-conjugate under a group of order $d, 2 d$, or $4 d$. For two of the fixed lines of the cyclic group must be the axes of a congruence contained by the complex and any transformation interchanging them must be of period 4 on the vertices of the invariant tetrahedron if no skew perspectivities of higher period than 2 appear. No transformations of a cyclic group can leave invariant the axes of a reflection unless all of them do, for otherwise there would be transformations leaving fixed the axes of more than one reflection. Also if two cyclic groups of odd order contain transformations in common, the group generated by them must contain an invariant reflection whose axes are axes of a congruence belonging to the complex. Hence there will be $\Omega / d, \Omega /(2 d)$, or $\Omega /(4 d)$ conjugate cyclic groups of order $d$ containing $(d-1) \Omega / d,(d-1) \Omega /(2 d)$, or $(d-1) \Omega /(4 d)$ transformations.

If we attempt to enumerate the transformations which the group must contain, we are led to the following diophantine equation:

$$
\Omega=1+\sum_{i=1}^{r}\left(d_{i}-1\right) \frac{\Omega}{f_{i} d_{i}},
$$

where $f_{1}=2, f_{i}=1,2,4$ for $i>1$. Also if $f_{i}=4, d_{i}$ can be divisible by no factors of the form $4 n-1$.

If no $f_{i}=4$, the solutions of this equation are well known.* They correspond to cyclic, dihedral, tetrahedral, octahedral, and icosahedral groups. All of these groups exist here, but the icosahedral group is the only one which is primitive.

* This equation was first discussed by Jordan, J o u rnal für die reine und a ngewand te Mathematik, vol. 84 (1878), p. 89. 
If one or more $f_{i}$ have the value 4 , it is found that $r=3, f_{1}=f_{2}=2$, $f_{3}=4$, since we may suppose that $d_{1}$ is divisible by 4 , and if $f_{i}=4, d_{i}=5$, 13,17 , etc.

Suppose first that $d_{1}=4$. Then $d_{2}=5,6$. If $d_{2}=5, d_{3} \nless 13$, which is impossible. If $d_{2}=6$, then $d_{3}=5, \Omega=120$.

Next suppose that $d_{1} \bar{\Sigma}$. Since $d_{3} \nless 5$, we must have $d_{2}<4$. If $d_{2}=3$, we must have $d_{3}=5, d_{1}=12$. But this is impossible since there can be but one conjugate set of $C_{3}$. We find that $d_{2}=2$ is impossible.

The solution $r=3, f_{1}=f_{2}=2, f_{3}=4, d_{1}=4, d_{2}=6, d_{3}=5, \Omega=120$, must represent a group which can be represented as a permutation group on the six $C_{5}$. It must therefore be isomorphic with the symmetric group on 5 letters. A group of this sort occurs' as a subgroup of the $G_{720}$ and it may be shown that there is only one type of such a group which is primitive and which leaves invariant a linear complex.

Theorem 15. The only primitive groups which contain no homologies and no skew perspectivities of higher period than 2 , but which contain a $G_{16}$ having no operator of higher period than 2 , leave invariant either that $G_{16}$ or another $G_{16}$ of the same type.

Consider the $G_{16}$ generated by $S:\left[-x_{1},-x_{2}, x_{3}, x_{4}\right], T:\left[x_{1},-x_{2}\right.$, $\left.-x_{3}, x_{4}\right], U:\left[x_{2},-x_{1},-x_{4}, x_{3}\right], V:\left[-x_{4},-x_{3}, x_{2}, x_{1}\right]$. Any group which contains this $G_{16}$, but does not leave it invariant, must contain more reflections commutative with some of the reflections in it. For consider a reflection not in the $G_{16}$. If its product by any one of the reflections in the $G_{16}$ is of even period it is either commutative with a reflection in the $G_{16}$ or is commutative with one which is. If its product by every one of the reflections in the $G_{16}$ is of odd period, it will be conjugate with all of them. If there were no more reflections commutative with any of those in the $G_{16}$, the $G_{16}$ would have to remain invariant under a group which was transitive on the 15 reflections. But in any such group the reflections in the $G_{16}$ are commutative with other reflections.

If there is an additional reflection interchanging the axes of a reflection in the $G_{16}$, it will either leave fixed the axes of a reflection in the $G_{16}$ or there will be other reflections leaving fixed the axes which it interchanges. Consider a reflection $W$ leaving fixed the axes of $S$. Since $V W$ will interchange the axes of $S,(V W)^{2}$ must be a reflection. Moreover $(V W)^{2}$ must be in the $G_{16}$, since it will be commutative with both $S$ and $V$. We suppose $(V W)^{2}$ $=S U$. Hence $W$ must interchange the two pairs of points, (1i00) and $(1-i 00),(00 i 1)$ and $(00-i 1)$. Since the vertices of this tetrahedron are interchanged by pairs in all three ways, it may be shown that no transformation of higher period than 4 can leave them all fixed without involving a skew perspectivity of higher period than 2 . Hence $(T W)^{4}=I$ and 
$(T U W)^{4}=I$. In order that no skew perspectivity of period 4 shall appear, $W$ must have the same fixed points as $T$ on one of the axes of $S$ and the same fixed points as $T U$ on the other. Four such reflections are possible, but if one is present all will be. One of them is $W:\left[x_{2}, x_{1}, x_{3},-x_{4}\right]$.

If a primitive group exists, $S$ must be conjugate with reflections which interchange its axes. For otherwise $S$ and $V$ could not be conjugate and hence all the reflections conjugate with $S$ would be commutative with reflections which were commutative with $V$. But we find it impossible to add reflections of this sort which are not conjugate with reflections interchanging their axes. Hence the only reflections with which $S$ can be conjugate are those leaving fixed its axes. No primitive group is therefore possible.

If $S$ is conjugate with reflections which interchange its axes, there will be a group leaving invariant a linear complex in which the axes of $S$ and the axes of a reflection interchanging its axes are axes of congruences. In order that no skew perspectivities of period higher than 2 shall be present, this group must be primitive and of order $16 \cdot 10$.

Consider then a group of order $16 \cdot 10$. We suppose that the invariant $G_{16}$ is that generated by $S, T, U, V$, and that the group of order $16 \cdot 10$ is generated by these together with $W$ and a reflection leaving fixed the axes of $V$, which we denote by

$X: \quad\left[\frac{1-i}{2}\left(x_{2}+x_{3}\right), \frac{1+i}{2}\left(x_{1}-x_{4}\right), \frac{1+i}{2}\left(x_{1}+x_{4}\right), \quad \frac{1-i}{2}\left(-x_{2}+x_{3}\right)\right]$.

It follows then that no more reflections can leave fixed the axes of any one of the five reflections in this group (such as $S$ or $V$ ) whose axes are axes of congruences belonging to the complex, since they would leave the complex invariant.

Under a larger group a reflection not in the invariant $G_{16}$ of the group of order 16 10 must be commutative with more reflections. Consider then a reflection $Y$ leaving fixed the axes of $X$. If it is commutative with either $T$ or $V$ it must be commutative with both. If it is not commutative with them $(T Y)^{2}$ must be a reflection commutative with $T$ and hence with $V$, since $T Y$ interchanges the axes of $X$. We may then suppose that $Y$ has this property. One of the reflections which must then be in the group is $Y:\left[x_{1}, x_{3}, x_{2},-x_{4}\right]$. A group of order $16 \cdot 60$ is then generated, permuting five tetrahedra, one of which is $(100 i)(100-i)(01 i 0)(01-i 0)$.

We may show that no group can contain the group of order $16 \cdot 60$. If this is the case more reflections must be commutative with the reflections which are not in the invariant $G_{16}$. A reflection $Z$ leaving fixed the axes of $Y$ cannot interchange the axes of $T$, for in that case it would have to leave fixed the axes of either $V$ or $T V$. Hence it must leave invariant (0001) and (01 - 10) 
and interchange (1000) and (0110), or vice versa. Consider a reflection of the first sort. The fixed points of $Z$ on the latter line must coincide with one of the two pairs of points in which the axes of $X$ and $T X$ meet that line, i. e. $(i-1,1,1,0)(-i+1,1,1,0)$, or $(i+1,1,1,0)(-i-1,1,1,0)$. But $S, T, W, Y, Z$ all leave fixed $(0001)$ and $x_{4}=0$ and must therefore generate a $G_{168}$ in that plane. But this is not the case unless the coefficients of the transformations are reduced modulo $3 .^{*}$

Theorem 16. The only primitive group which contains reflections which interchange each other's axes, but which does not leave a linear complex invariant, does not contain $a G_{16}$ in which there is no operator of higher period than 2 , and does not contain any skew perspectivities of higher period than $\mathcal{Z}$ or any homologies is a $G_{168}$.

Two reflections which interchange each other's axes and a third reflection not commutative with any one of the three in the four-group generated by them must generate a group leaving a complex invariant, i. e., the complex determined by the axes of the three generating reflections. If this group is primitive, it must be either a $G_{120}$ or a $G_{60}$. If it is imprimitive, it must interchange two lines of the complex and will be either an octahedral group or a group of order $48,(2,1)$ isomorphic with an octahedral group.

We will show that no group of the type we are seeking can contain either a $G_{120}$ or a $G_{60}$. There can be no reflection leaving fixed both axes of a reflection which belongs to either of these groups. For either this reflection would be commutative with a four-group of the $G_{120}$ or $G_{60}$ containing that reflection or else the square of its product by a reflection interchanging the axes which it leaves fixed would be such a reflection. But a reflection leaving fixed the axes of all three reflections in a four-group which is invariant under a tetrahedral group must remain invariant under the tetrahedral group, for otherwise there would be either a $G_{16}$ containing no $C_{4}$ or a skew perspectivity of higher period than 2 leaving that four-group invariant. The axes of this reflection

* The fixed points of $Z$ on the line joining ( 1000$)$ to $(0110)$ must be $( \pm \lambda, 1,1,0)$, where $\lambda^{2}-\lambda+2=0$. This is true of either pair of points in case the coordinates are reduced modulo 3 . In this case a group of order 20160 is generated, permuting 120 points conjugate with ( 0001 ). One of the reflections which leaves fixed the axes of $W$ we take to be Z: $\quad\left[(1-i)\left(x_{2}+x_{3}\right),(1+i) x_{1}+x_{2}-x_{2},(1+i) x_{1}-x_{2}+x_{2}, x_{4}\right]$.

This group contains two conjugate sets of subgroups of order $16 \cdot 60$. The 21 subgroups in each conjugate set arrange themselves by fives in 21 ways. For there are associated with one such group five groups not conjugate with it. The $G_{1 a .00}$ generated by $S, T, U, V, W$, $X, Y$ contains five $G_{10}$ not conjugate with its invariant $G_{10}$, one of which is generated by $T, V, X, Y$. Any two of these five $G_{10}$ generate the whole group of order $16 \cdot 60$. It may readily be shown that all such arrangements of 21 letters are conjugate under the symmetric group on those letters. Hence the group of order 20160 is $(1,1)$ isomorphic with the group of all ternary transformations with coefficients in the $G F\left(2^{2}\right)$ and determinant unity. It

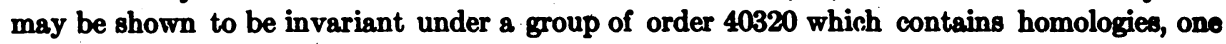
of these being $\left[x_{1}, x_{3}, x_{3},-x_{4}\right]$. 
must therefore be the two fixed lines of the tetrahedral group. But these lines are lines of the complex and hence the reflection would leave the complex invariant. But this is impossible.

Since a $G_{120}$ contains two conjugate sets of reflections, a larger group containing it must contain more reflections commutative with any reflection in at least one of the two conjugate sets. Such a reflection would leave a complex invariant in common with the dihedral group in the $G_{120}$ which leaves invariant the reflection with which it is commutative. It must then generate with that dihedral group a group leaving a regulus invariant. But there must then be reflections which leave fixed the axes of the invariant reflection.

Consider now a $G_{60}$. A reflection not in the $G_{60}$ and which is not commutative with any one of the three reflections of a four-group of the $G_{\infty 0}$ must generate with that four-group either a $G_{60}$ or a $G_{24}$. If it is a $G_{80}$ it must contain the same $G_{12}$ leaving the four-group invariant, for otherwise there would be skew perspectivities commutative with that four-group. But it would then leave invariant the same complex, since the $G_{12}$ leaves invariant only one complex. If it is a $G_{24}$, the four-group would be invariant under a $G_{8}$. This $G_{8}$ would have to leave invariant the $G_{12}$ and hence the complex. But this is impossible, since we can have no $G_{120}$.

We inquire whether any primitive group of the type we are seeking can contain a $G_{48}$, which is $(2,1)$ isomorphic with a $G_{24}$. The $G_{48}$ contains twelve reflections which interchange the axes of the invariant reflection. In a primitive group containing the $G_{48}$ there must be more reflections interchanging the axes of these reflections. Such a reflection will leave a complex invariant in common with a dihedral $G_{12}$ in the $G_{48}$ which contains the reflection with which it is commutative. It must generate with that $G_{12}$ a group which is primitive. But we have considered all such cases.

We suppose finally that any four-group in which each reflection interchanges the axes of the other two and another reflection not commutative with any one of the three in the four-group generate a $G_{24}$. A reflection not in a particular $G_{24}$ must generate with the invariant four-group of that $G_{24}$ another $G_{24}$. That four-group can be left invariant only by the other three four-groups of the original $G_{24}$. Hence this four-group can lie in but three $G_{24}$ other than the one under which it remains invariant, i. e., $G_{24}$ having for invariant fourgroups the other three four-groups of the original $G_{24}$. There must be exactly two more reflections commutative with each reflection of the original $G_{24}$ which does not lie in the invariant four-group. There must then be $3+6$ $+6 \cdot 2$ reflections and the group must be of order $21 \cdot 8=168$.

A $G_{168}$ occurs as a subgroup of the $G_{2520}$ and it may be shown that there is only one type of such a group which is primitive.*

\footnotetext{
* Cf. Bucheredr, M a t h e mat i s c h e A n n a le n, vol. 60 (1905), p. 226.
} 
§ 7. Groups containing no skew reflections which interchange each other's axes.

Theorem 17. The only primitive group, which contains no homologies, no skew perspectivities of higher period than 2 , and no reflections interchanging each other's axes, but which contains reflections leaving fixed each other's axcs and reflections whose axes met along a single line, is a $G_{60}$ leaving a quadric invariant.

It may readily be shown that two reflections whose axes meet along a single line and a third reflection leaving fixed each of the axes of one of the reflections in the group generated by the first two leave at least one quadric invariant.

Any group which can be generated by two reflections whose axes meet along a single line will be contained by a group leaving a quadric invariant, since any one of the reflections in the dihedral group will be commutative with reflections which leave fixed its axes and which do not leave the dihedral group invariant. If the product of the two reflections is of period greater than 5 , no such group is possible. If the product is of period 5, the only possible group is a $G_{60}$ leaving invariant a point and a plane and a pencil of quadrics. If the product is of period 4 , the only possible group is a $G_{24}$ leaving invariant a point and a plane and a pencil of quadrics. If the product is of period 3 , the group may be of either the two previous types or a $G_{60}$ permuting five points.

Consider a $G_{60}$ leaving invariant a point and a plane and a pencil of quadrics. We inquire whether any group can contain such a $G_{60}$. There can be no more reflections transforming a $C_{3}$ or a $C_{5}$ into its inverse, since such a reflection would leave invariant one of the quadrics of the family left invariant by the $G_{60}$. Hence if $\Omega$ denote the order of the group containing the $G_{60}$ it must contain $(3-1) \Omega /(2 \cdot 3)$ transformations of period 3 and $(5-1) \Omega /(2 \cdot 5)$ transformations of period 5 . We find that there can be no more reflections commutative with a reflection in the $G_{60}$, since every such reflection would have to generate another $G_{60}$ with each of the two $G_{10}$ containing the reflection with which it is commutative. A four-group will be invariant either under a group of order 48 transitive on the vertices of its invariant tetrahedron or merely under a tetrahedral group and there will be either $(16-1) \Omega / 48$ or $(4-1) \Omega / 12$ transformations commutative with the four-groups. The former is evidently impossible, since the number of transformations would exceed $\Omega$. In the latter case we must have

$$
\Omega=1+(4-1) \frac{\Omega}{12}+(3-1) \frac{\Omega}{2 \cdot 3}+(5-1) \frac{\Omega}{2 \cdot 5}+\cdots .
$$

No other terms representing transformations can be added and we have $\Omega=60$. Hence no group can contain the $G_{60}$.

Consider now a $G_{24}$ leaving fixed a point and a plane and a pencil of quadrics. In any larger group a reflection not in the invariant four-group must be com- 
mutative with more reflections. Any such reflection must generate together with the $G_{8}$ which contains the reflection with which it is commutative another $G_{24}$. Such a reflection will either leave invariant one quadric of the family left invariant by the original $G_{24}$ or will leave invariant the fixed point and plane of that $G_{24}$. In the latter case we must have a $G_{168}$.

No group can contain the $G_{168}$. For no more reflections can be commutative with a reflection in that group or can transform a $C_{3}$ into its inverse without leaving invariant a quadric which remains invariant under a $G_{24}$ of the $G_{168}$. Also a $C_{7}$ can be self-conjugate under no larger group than a $G_{21}$. Hence if $\Omega$ denote the order of the whole group, we must have

$$
\Omega=1+(4-1) \frac{\Omega}{2 \cdot 4}+(3-1) \frac{\Omega}{2 \cdot 3}+(7-1) \frac{\Omega}{3 \cdot 7}+\cdots .
$$

No more terms representing transformations can be added and we have $\Omega=168$. Hence no group can contain the $G_{168}$.

Consider now a $G_{60}$ permuting five points. We may now suppose that the product of any two reflections whose axes meet along a single line is of period 3. There can be no more reflections transforming a $C_{3}$ into its inverse. For such a reflection would have to generate another $G_{60}$ with a $G_{12}$ containing the $C_{8}$ and hence its product by one of the reflections in the invariant four-group of the $G_{12}$ would be of period 3. But its axes would then meet the fixed line of the $C_{3}$ which does not intersect the line of fixed points in the same points in which that line is met by the axes of a reflection in the original $G_{60}$. The product of these two reflections would have to be of period 3. We would then have two commutative $C_{3}$, which is impossible.

A reflection can lie in but one four-group. For such a four-group would generate with any $G_{6}$ containing the reflection of the four-group belonging to the original $G_{60}$ another $G_{60}$. But all $G_{60}$ must be conjugate, since all fourgroups must be conjugate, all tetrahedral groups containing a four-group must be conjugate, and a tetrahedral group can be contained by only one $G_{60}$, as a $C_{3}$ can be contained by only one $G_{6}$. The two $G_{60}$ having the $G_{6}$ in common would then have to be conjugate under a group leaving the $G_{6}$ invariant. But this would involve an impossible group commutative with a reflection.

A $C_{5}$ can be invariant only under a $G_{10}$. For there can be no $C_{4}$ permuting the vertices of its invariant tetrahedron, since such a $C_{4}$ would have to leave invariant the four-group commutative with its square and would hence leave the $G_{60}$ invariant. But the only group containing the $G_{60}$ self-conjugately is a $G_{120}$ which contains homologies of period 2. Also if the $C_{5}$ is invariant under an abelian group of higher order, there must be additional reflections leaving the quadric invariant. 
There can be no transformation leaving invariant one reflection of a fourgroup and interchanging the other two, since in that case a tetrahedral group would be invariant under a $G_{24}$. Let $d_{1}$ denote the order of the group commutative with a four-group and let $\Omega$ denote the order of the whole group. Then we must have

$$
\Omega=1+\left(d_{1}-1\right) \frac{\Omega}{3 d_{1}}+(3-1) \frac{\Omega}{2 \cdot 3}+(5-1) \frac{\Omega}{2 \cdot 5}+\cdots .
$$

We must evidently have $d_{1}=4$ and no more terms representing transformations can be added. Then $\Omega=60$. Hence no group can contain the $G_{80}$.

Theorem 18. There is no primitive group which contains no homologies, no skew perspectivities of higher period than 2 , no reflections interchanging each other's axes, and no reflections whose axes meet along a single line, but which contains reflections leaving fixed each other's axes.

It may be shown that in any group of the sort described above at least two of the reflections in a four-group must be conjugate. For any reflection will certainly be conjugate with more than one-third of the reflections in the group, the only reflections with which it may not be conjugate being those commutative with it and some of the reflections commutative with the latter.

If a reflection is conjugate with one commutative with it there will be transformations sending one to the other and leaving invariant the fourgroup generated by the two, since all four-groups commutative with a reflection must be conjugate. Such a transformation will either leave invariant the third reflection of the four-group or will permute the three cyclically.

We consider the first case. The transformation cannot be of period 2 or 4 , since there would then be reflections whose axes met along a single line. Hence it must be of higher period than 4 . But then the reflection with which it is commutative can lie in but the one four-group. If a primitive group exists, all reflections must then be conjugate. Hence all six permutations will be made on the three reflections of the four-group. But in such a case any tetrahedral group containing the four-group would be invariant under an octahedral group and hence there would be reflections whose axes met along a single line.

We now suppose that a transformation which leaves a four-group invariant and is not commutative with all three reflections permutes the three cyclically. No transformation except the identity can leave more than one $C_{3}$ invariant, since we can have no reflections whose axes intersect along a single line. Hence if $\Omega$ denotes the order of the whole group and $d_{1}$ the order of the group leaving a $C_{3}$ invariant, there must be $\Omega / d_{1}$ such groups and $\left(d_{1}-1\right) \Omega / d_{1}$ transformations in them.

Suppose first that a reflection lies in only one four-group. If the order of 
the group commutative with a four-group be denoted by $d_{2}$, there must be $\Omega /\left(3 d_{2}\right)$ four-groups and $\left(d_{2}-1\right) \Omega /\left(3 d_{2}\right)$ transformations commutative with them. We should then have

$$
\Omega=1+\left(d_{1}-1\right) \frac{\Omega}{d_{1}}+\left(d_{2}-1\right) \frac{\Omega}{3 d_{2}}+\cdots .
$$

We must have $d_{1}=3$ and no more terms representing transformations can be added. Then $\Omega=3 d_{2}$.

Suppose now that a reflection lies in more than one four-group. If the order of the cyclic group commutative with a reflection be denoted by $d_{2}$, there must be either $\Omega /\left(2 d_{2}\right)$ reflections and $\left(d_{2}-1\right) \Omega /\left(2 d_{2}\right)$ transformations commutative with them or else $\Omega /\left(4 d_{2}\right)$ reflections and $\left(3 d_{2} / 2-1\right) \Omega /\left(4 d_{2}\right)$ transformations commutative with them. (In the latter case one-half the transformations interchanging the vertices of the invariant tetrahedron by pairs will be of period 4.) But either case is impossible, since the number of transformations in the group would exceed $\Omega$.

Theorem 19. There is no primitive group containing no homologies, no skew perspectivities of higher period than 2 , and no four-groups of either type.

No transformation other than the identity can leave more than one reflection invariant unless the axes of those reflections intersect along a single line and the transformation leaves fixed all the points on the other fixed line of the dihedral group generated by the reflections. In this case one-half of the transformations which leave a reflection invariant would leave no other reflections invariant. The abelian group of order $d_{1}$ contained by this group will be self-conjugate either under the group of order $2 d_{1}$, or under a group of order $6 d_{1}$. In the latter case there must be also an abelian group whose order $\left(d_{2}\right)$ is divisible by 3 and which can be self-conjugate only under a group of order $2 d_{2}$. Hence the order $(\Omega)$ of the group which contains transformations leaving invariant more than one reflection must satisfy a diophantine equation of one of the two types:

$$
\begin{aligned}
& \Omega=1+\frac{1}{2} \Omega+\left(d_{1}-1\right) \frac{\Omega}{2 d_{1}}+\cdots ; \\
& \Omega=1+\frac{1}{2} \Omega+\left(d_{1}-1\right) \frac{\Omega}{6 d_{1}}+\left(d_{2}-1\right) \frac{\Omega}{2 d_{2}}+\cdots
\end{aligned}
$$

In neither case can more terms representing transformations be added. In the first case we have $\Omega=2 d_{1}$. In the second case we must have $d_{2}=3$, $\Omega=6 d_{1}$.

In case there is no transformation except the identity leaving invariant more than one reflection, we find readily, by similarly constructed equations 
that either there is but one reflection, or there is a dihedral group in which each reflection is commutative with itself only, or there is a group permuting cyclically the four vertices of a tetrahedron, in which each reflection is commutative with a $C_{4}$.

In case there are no reflections at all, we find readily that there is either an abelian group, or a group of order $3 d$, where $d$ denotes the order of an abelian group.

University of Pennsylvania. 